A NNALES

UNIVERSITATIS MARIAE CURIE-SKŁODOWSKA

LUBLIN - POLONIA

VOL. LXVIII, NO. 1, 2014

SECTIO A

$43-47$

\author{
T. D. NARANG
}

\title{
A fixed point theorem for nonexpansive compact self-mapping
}

\begin{abstract}
A mapping $T$ from a topological space $X$ to a topological space $\mathrm{Y}$ is said to be compact if $T(X)$ is contained in a compact subset of $Y$. The aim of this paper is to prove the existence of fixed points of a nonexpansive compact self-mapping defined on a closed subset having a contractive jointly continuous family when the underlying space is a metric space. The proved result generalizes and extends several known results on the subject.
\end{abstract}

Finding sufficient conditions for the existence of fixed points of a selfmapping $f$ defined on a topological space $X$ is an interesting aspect of the theory of fixed points. Several results are known in the literature on this subject. In 1930, J. Schauder [11] proved that if $K$ is a compact convex subset of a Banach space $X$ and $T: K \rightarrow K$ is a continuous map, then $T$ has at least one fixed point in $K$. The compactness condition on $K$ is a strong one. Many problems in analysis do not have a compact setting and the spaces are not Banach spaces. So, it is natural to prove the result by relaxing the condition of compactness and considering spaces more general than Banach spaces. In this direction many results are available in the literature (see e.g. [1]-[4], [6]-[8] and [10]). In this paper we also prove a result on the existence of fixed points of a nonexpansive compact selfmapping defined on a closed subset having a contractive jointly continuous

2000 Mathematics Subject Classification. 47H10, 54H25.

Key words and phrases. Convex metric space, convex set, star-shaped set, nonexpansive map, compact map.

The research has been sponsored by University Grants Commission, India under Emeritus Fellowship. 
family when the underlying space is a metric space. The proved result generalizes and extends some of the results proved in [1], [2]-[4], [7]-[9].

To start with, we recall few definitions and some related results.

Let $X$ and $Y$ be topological spaces. A mapping $T: X \rightarrow Y$ is called compact if $T(X)$ is contained in a compact subset of $Y$.

If $T$ is compact, then $\overline{T(X)}$ is compact.

Let $(X, d)$ be a metric space. A continuous mapping $W: X \times X \times[0,1] \rightarrow$ $X$ is said to be a convex structure on $X$ if for all $x, y \in X$ and $\lambda \in[0,1]$,

$$
d(u, W(x, y, \lambda)) \leq \lambda d(u, x)+(1-\lambda) d(u, y)
$$

holds for all $u \in X$. The metric space $(X, d)$ together with a convex structure is called a convex metric space [12].

A subset $K$ of a convex metric space $(X, d)$ is said to be a convex set [12] if $W(x, y, \lambda) \in K$ for all $x, y \in K$ and $\lambda \in[0,1]$. The set $K$ is said to be $\boldsymbol{p}$-starshaped [8] if there exists a $p \in K$ such that $W(x, p, \lambda) \in K$ for all $x \in K$ and $\lambda \in[0,1]$. Clearly, each convex set is starshaped but not conversely.

A convex metric space $(X, d)$ is said to satisfy property (I) [8] if for all $x, y, q \in X$ and $\lambda \in[0,1]$,

$$
d(W(x, q, \lambda), W(y, q, \lambda)) \leq \lambda d(x, y) .
$$

A normed linear space and each of its convex subsets are simple examples of convex metric spaces. There are many convex metric spaces which are not normed linear spaces (see e.g. [8], [12]). Property (I) is always satisfied in a normed linear space.

Let $K$ be a subset of a metric space $(X, d)$ and $\mathfrak{F}=\left\{f_{\alpha}: \alpha \in K\right\}$ a family of functions from $[0,1]$ into $K$, having the property $f_{\alpha}(1)=\alpha$ for each $\alpha \in K$. Such a family $\mathfrak{F}$ is said to be

(i) contractive if there exists a function $\phi:(0,1) \rightarrow(0,1)$ such that for all $\alpha, \beta \in K$ and for $t \in(0,1)$, we have

$$
d\left(f_{\alpha}(t), f_{\beta}(t)\right) \leq \phi(t) d(\alpha, \beta),
$$

(ii) jointly continuous if $t \rightarrow t_{0}$ in $[0,1]$ and $\alpha \rightarrow \alpha_{0}$ in $K$ imply $f_{\alpha}(t) \rightarrow$ $f_{\alpha_{0}}\left(t_{0}\right)$.

In normed linear spaces these notions were discussed by Dotson [5].

If $K$ is a starshaped subset with star centre $q$ of a convex metric space $(X, d)$ with property $(\mathrm{I})$, then the family $\mathfrak{F}=\left\{f_{x}: x \in K\right\}$ defined by $f_{x}(t)=W(x, q, t)$ satisfies

$$
d\left(f_{x}(t), f_{y}(t)\right)=d(W(x, q, t), W(y, q, t)) \leq t d(x, y) .
$$

So taking $\phi(t)=t, 0<t<1$, the family $\mathfrak{F}$ is a contractive jointly continuous family and therefore the class of subsets of $X$ with the property of contractiveness and joint continuity contains the class of starshaped sets which in turn contains the class of convex sets. 
Considering the existence of fixed points for nonexpansive mappings in convex metric spaces, Beg and Abbas [2] proved that if $K$ is a nonempty compact and convex subset of a complete convex metric space $(X, d)$ with property (I), then any nonexpansive mapping $T: K \rightarrow K$ has a fixed point. This result is not true in general for noncompact sets $K$ (see [7], p. 12). Various extensions and generalizations of the results of Schauder [11] and of Beg and Abbas [2] are known in the literature (see e.g. [1], [3], [4], [6]-[10]). In this direction, we also prove the following:

Theorem 1. Let $(X, d)$ be a metric space and $K$ a non-empty closed subset with a contractive jointly continuous family $\left\{f_{\alpha}: \alpha \in K\right\}$. If $T: K \rightarrow K$ is a nonexpansive compact mapping, then $T$ has a fixed point.

Proof. Let $k_{n}=\frac{n}{n+1}, n=1,2,3, \ldots$ Define $T_{n}: K \rightarrow K$ as

$$
T_{n} x=f_{T x}\left(k_{n}\right), x \in K .
$$

Since $T(K) \subset K$ and $k_{n}<1, T_{n}: K \rightarrow K$ is well defined. Consider

$$
\begin{aligned}
d\left(T_{n} x, T_{n} y\right) & =d\left(f_{T x}\left(k_{n}\right), f_{T y}\left(k_{n}\right)\right) \\
& \leq \phi\left(k_{n}\right) d(T x, T y) \\
& \leq \phi\left(k_{n}\right) d(x, y), x, y \in K
\end{aligned}
$$

and so each $T_{n}$ is a contraction mapping on $K$. Since $c l T_{n}(K)$ is $T_{n^{-}}$ invariant and also compact for each $n$ and hence complete, by Banach contraction principle, each $T_{n}$ has a unique fixed point $x_{n} \in K$ i.e. $T_{n} x_{n}=x_{n}$.

Since $T(K)$ lies in a compact subset of $K,\left(T x_{n}\right)$ has a subsequence $\left(T x_{n_{i}}\right)$ such that $\left(T x_{n_{i}}\right) \rightarrow x_{0} \in K$.

Now $x_{n_{i}}=T_{n_{i}}\left(x_{n_{i}}\right)=f_{T x_{n_{i}}}\left(k_{n_{i}}\right) \rightarrow f_{x_{0}}(1)=x_{0}$. Since $T$ is a continuous, $\left(T x_{n_{i}}\right) \rightarrow T x_{0}$ and hence $x_{0}=T x_{0}$ i.e. $x_{0}$ is a fixed point of $T$.

If $K$ is a starshaped subset with a star centre $p$, of a convex metric space $(X, d)$ with property (I), then the family $\left\{f_{\alpha}: \alpha \in K\right\}$ defined by $f_{\alpha}(t)=W(\alpha, p, t)$ is contractive if we take $\phi(t)=t$ for $0<t<1$, and is jointly continuous. So, we have

Corollary $1([3])$. Let $(X, d)$ be a convex metric space with property (I) and $K$ a non-empty closed starshaped subset of $X$. If $T: K \rightarrow K$ is a nonexpansive compact map, then $T$ has a fixed point.

Since every normed linear space is a convex metric with property (I), as an immediate consequence of Corollary 1, we get:

Corollary 2 ([9]). If $T: K \rightarrow K$ is a nonexpansive, where $K$ is a closed and starshaped subset of a normed linear space $E$, and $c l[T(K)]$ is compact, then $T$ has a fixed point.

Since every convex set is starshaped, we have 
Corollary 3. Let $K$ be a non-empty closed convex subset of a convex metric space $(X, d)$ with property $(I)$, then any nonexpansive compact mapping $T$ : $K \rightarrow K$ has a fixed point.

Since for a compact set $K$, any continuous mapping $T: K \rightarrow K$ is a compact mapping, we have

Corollary $4([2])$. Let $K$ be a compact subset of a complete convex metric space $(X, d)$. Suppose there exists a contractive, jointly continuous family of maps associated with $K$, then any nonexpansive mapping $T$ of $K$ into itself has a fixed point in $K$.

Note. We do not require the completeness of the space in Corollary 4 proved by Beg and Abbas [2].

Corollary 5 ([2]). Let $K$ be a non-empty compact convex (or starshaped) subset of a convex metric space $(X, d)$ with property $(I)$, then any nonexpansive mapping $T: K \rightarrow K$ has a fixed point.

Corollary $6([8])$. Let $X$ be a compact starshaped metric space satisfying property (I). Let $T: X \rightarrow X$ be a nonexpansive mapping. Then $T$ has a fixed point in $X$.

Since every normed linear space is a convex metric space with property (I), we have

Corollary 7 ([7], Theorem 3.1, p. 28). If $K$ is a non-empty compact and convex subset of a Banach space, then any nonexpansive mapping $T: K \rightarrow$ $K$ has a fixed point.

Corollary 8 ([1]). Let $K$ be a closed starshaped or convex subset of a Banach space $X$ and $f: K \rightarrow K$ a nonexpansive map with $f(K)$ contained in a compact set $K$. Then $f$ has a fixed point.

Corollary 9 ([4]). Let $X$ be a Banach space and $K$ be a nonempty compact starshaped subset of $X$. Let $T: K \rightarrow K$ be a nonexpansive mapping. Then $T$ has a fixed point in $K$.

Acknowledgement. The author is thankful to the learned referee for the critical comments and valuable suggestions leading to an improvement of the paper.

\section{REFERENCES}

[1] Agarwal, R. P., Meehan, M., O'Regan, D., Fixed Point Theory and Applications, Cambridge University Press, Cambridge, 2001.

[2] Beg, I., Abbas, M., Fixed points and best approximation in Menger convex metric spaces, Arch. Math. (Brno) 41 (2005), 389-397.

[3] Beg, I., Shahzad, N., Iqbal, M., Fixed point theorems and best approximation in convex metric spaces, J. Approx. Theory 8 (1992), 97-105. 
[4] Dotson Jr., W. G., Fixed-point theorems for nonexpansive mappings on starshaped subset of Banach spaces, J. London Math. Soc. 2 (1972), 408-410.

[5] Dotson Jr., W. G., On fixed points of nonexpansive mappings in nonconvex sets, Proc. Amer. Math. Soc. 38 (1973), 155-156.

[6] Dugundji, J., Granas, A., Fixed Point Theory, PWN-Polish Sci. Publ., Warszawa, 1982 .

[7] Goebel, K., Kirk, W. A., Topics in Metric Fixed Point Theory, Cambridge University Press, Cambridge, 1990.

[8] Guay, M. D., Singh, K.L., Whitfield, J. H. M., Fixed point theorems for nonexpansive mappings in convex metric spaces, Proc. Conference on nonlinear analysis (Ed. S.P. Singh and J.H. Bury), Marcel Dekker, New York, 1982, 179-189.

[9] Habiniak, L., Fixed point theory and invariant approximations, J. Approx. Theory 56 (1989), 241-244.

[10] Singh, S., Watson, B., Srivastava, P., Fixed Point Theory and Best Approximation: The KKM-map Principle, Kluwer Academic Publishers, Dordrecht, 1997.

[11] Schauder, J., Der fixpunktsatz in funktionaraumen, Studia Math. 2 (1930), 171-180.

[12] Takahashi, W., A convexity in metric space and nonexpansive mappings, I, Kodai Math. Sem. Rep. 22 (1970), 142-149.

T. D. Narang

Department of Mathematics Guru

Nanak Dev University

Amritsar -143005

India

e-mail: tdnarang1948@yahoo.co.in

Received October 11, 2011 